\title{
The PHI2 method: a way to compute time-variant reliability
}

\author{
C. Andrieu-Renaud ${ }^{\mathrm{a}, *}$, B. Sudret ${ }^{\mathrm{b}, 1}$, M. Lemaire ${ }^{\mathrm{a}}$ \\ ${ }^{a}$ LaRAMA-IFMA and UBP, Campus de Clermont-Ferrand/les Cézeaux, BP 265 F-63175 Aubière Cedex, France \\ ${ }^{\mathrm{b}}$ EDF R\&D, Site des Renardières-MMC, F-77818 Moret-sur-Loing Cedex, France
}

\begin{abstract}
Time-variant reliability problems appear in the engineering practice when (a) the material properties of the structure deteriorate in time or (b) random loading modelled as random processes is involved. This paper presents a method called PHI2 which is based on the outcrossing approach and allows to solve such problems using classical time-invariant reliability tools such as FORM/SORM methods. The PHI2 method is first presented. Then it is benchmarked with the well-established 'asymptotic methods' [Stochast. Process. Appl. 13 (1988) 195; J. Offshore Mech. Arctic Engng 113 (1991) 241; Probab. Engng Mech. 10 (1995) 53; J. Struct. Engng 25 (1998) 1] on three examples dealing with scalar or vector processes and linear or non-linear limit state functions. The PHI2 method appears more accurate in all cases. As an application example, the method is finally applied on a case representing a mechanical system (a beam) placed in an environment that can have exceptional configuration.
\end{abstract}

(C) 2003 Elsevier Ltd. All rights reserved.

Keywords: Time-variant reliability; Time-invariant reliability; FORM/SORM; Outcrossing rate; PHI2 method; Asymptotic approach

\section{Introduction}

Structural reliability analysis aims at computing the probability of failure of a mechanical system with respect to a prescribed failure criterion by accounting for uncertainties arising in the model description (geometry, material properties) or the environment (loading). In the context of durability analysis, i.e. the assessment of the structure all along its lifetime, it allows to compute the evolution of the reliability in time.

The time dependency appearing in these so-called timevariant reliability problems may be of two kinds:

- Material properties may be decaying in time. The degradation mechanisms usually present an initiation phase and a propagation phase. Both the initiation time and the propagation kinetics may be considered as random in the analyses. Examples of these mechanisms are fatigue crack growth in fracture mechanics, corrosion in steel structures or in reinforced concrete rebars, concrete shrinkage and creep phenomena, etc.

\footnotetext{
* Corresponding author. Tel.: +33-4-73-28-80-11; fax: + 33-4-73-28-8100 .

E-mail address: celine.andrieu@ifma.fr (C. Andrieu-Renaud).

${ }^{1}$ Tel.: +33-1-60-73-77-48; fax: +33-1-60-73-65-59.
}

- Loading may be randomly varying in time: in this case, stochastic processes are introduced in the analysis. This allows to account for environmental loading such as wind velocity, temperature, wave height, occupancy loads, traffic loads, etc.

When only degradation mechanisms are considered, the randomness is often represented by random variables multiplied by deterministic functions of time (the 'shape' of the degradation kinetics), leading to non-ergodic processes (i.e. their probabilistic description cannot be derived from a single trajectory). As these deterioration processes are usually monotonous in time (the crack length increases in time, the size of uncorroded zones decreases in time, etc.), it is possible to focus onto the end of the lifetime of the structure ('right-boundary problem'). In this case the problem may be solved using timeinvariant reliability tools.

The second kind of problems has been addressed by many authors in the past two decades, especially Breitung [1], Schall et al. [2], Engelung et al. [3], Rackwitz [4] using the so-called outcrossing approach. The probability of failure is related to the mean number of outcrossings of the random process through the limit state surface, either through estimation when the outcrossings may be considered as independent and Poisson distributed or through an upper bound in the general case. The approach, referred 
to as asymptotic method (AsM) in the sequel, couples analytical results for outcrossings of random processes with asymptotic integration.

Hagen and Tvedt [5] suggested another approach based on a parallel system reliability formulation for computing the outcrossing rate (as defined in Section 2). Li and Der Kiureghian [6,7], Der Kiureghian and Li [8] and Der Kiureghian [9] applied it to the study of a variety of reliability problems such as non-linear dynamics and inelastic space-variant finite element analysis. Vijalapura et al. [10] applied it to the study of the Bouc-Wen single degree-of-freedom hysteretic oscillator.

The PHI2 method presented in this paper [11-14] is inspired by this approach. It allows to address in a similar way all kinds of time-dependent problems, whatever the time dependency mentioned above.

After recalling the basics of time-variant reliability, the PHI2 method is presented. It is then compared to the classical asymptotic approach as implemented in the software COMREL-TV V7.1 [15]. The quality of the upper bound obtained by the PHI2 method is assessed by Monte-Carlo simulation. Finally, one illustrative example, dealing with concrete creep and random loading, is presented.

\section{Time-variant reliability concepts}

\subsection{Problem statement and notation}

Let us denote by $\mathbf{X}(t, \omega)$ the set of the random variables $X_{j}(\omega), j=1, \ldots, p$ and the one-dimensional random processes $X_{k}(t, \omega), k=p+1, \ldots, p+q$ describing the randomness in the mechanical problem under consideration. In this notation, $\omega$ stands for the outcome in the space of outcomes $\Omega$. The time-dependent limit state function associated with the reliability analysis is denoted by $G(t, \mathbf{X}(t, \omega))$. Positive values of $G(t, \mathbf{X}(t, \omega))$ correspond to the safe domain and negative or zero values to the failure domain. Zero values of $G(t, \mathbf{X}(t, \omega))$ define the limit state surface.

The failure of the structure within the time interval $[0, t]$ is represented by the event:

$$
E=\{\exists \tau \in[0, t] \mid G(\tau, \mathbf{X}(\tau, \omega)) \leq 0\}
$$

The (cumulative) probability of failure $P_{f, c}(0, t)$ of the structure within the interval $[0, t]$ is defined by:

$$
P_{f, c}(0, t)=\operatorname{Prob}(E)=\operatorname{Prob}(\exists \tau \in[0, t] \mid G(\tau, \mathbf{X}(\tau, \omega)) \leq 0)
$$

Let us define the so-called point-in-time (or instantaneous) probability of failure $P_{f, i}(t)$ at time $t$ by:

$$
P_{f, i}(t)=\operatorname{Prob}(G(t, \mathbf{X}(t, \omega)) \leq 0)
$$

This quantity is computed by considering time as a fixed parameter in Eq. (3) and by replacing the random processes by the corresponding random variables. Note that this probability does not take into account what happened before $t$. It is independent of the previous states, i.e. it is not a conditional probability. It will be used in the following as a computational intermediate.

\subsection{Case of decreasing limit state functions}

When only degradation phenomena are considered and no random processes are present in the problem statement, the limit state function $G$ usually depends on random variables and functions of time. Thus it may be written as $G(t, \mathbf{X}(\omega))$. Moreover, it is usually monotonously decreasing in time, meaning that all trajectories $G\left(t, \mathbf{X}\left(\omega_{0}\right)\right)$ (i.e. deterministic functions of time obtained from realizations of the random variables) are decreasing. If this property holds, the computation of $P_{f, c}(0, t)$ as defined in Eq. (2) reduces to that of $P_{f, i}(t)$ as defined in Eq. (3). Indeed:

$$
\begin{aligned}
P_{f, c}(0, t) & =1-\operatorname{Prob}[G(\tau, \mathbf{X}(\omega))>0 \quad \forall \tau \in[0, t]] \\
& =1-\operatorname{Prob}[G(t, \mathbf{X}(\omega))>0]=P_{f, i}(t)
\end{aligned}
$$

It is emphasized that this result only holds when the decrease of $G$ can be proven whatever the realizations of the random variables entering its definition. Assuming $G$ is continuously differentiable, this can be done by checking if $\mathrm{d} G / \mathrm{d} t \leq 0$, either analytically or numerically.

\subsection{The outcrossing approach}

When random loading and corresponding random processes are considered, no such simplified result can be derived. The usual approach to address the issue is the socalled outcrossing approach. Denoting by $N^{+}(0, t)$ the number of crossings from the safe state to the unsafe state in ] $0, t]$, one can write:

$P_{f, c}(0, t)=\operatorname{Prob}\left(\{G(0, \mathbf{X}(0, \omega)) \leq 0\} \cup\left\{N^{+}(0, t)>0\right\}\right)$

The latter equation may be interpreted as follows: failure within time interval $[0, t]$ corresponds either to failure at the initial instant $t=0$ or to a later outcrossing of the limit state surface if the system is in the safe domain at $t=0$. Classical arguments [16,17] lead to the following bounds on $P_{f, c}(0, t)$ :

$\max _{0 \leq \tau \leq t}\left(P_{f, i}(\tau)\right) \leq P_{f, c}(0, t) \leq P_{f, i}(0)+E\left[N^{+}(0, t)\right]$

The outcrossing rate $\nu^{+}(\tau)$ is defined as the following limit:

$\nu^{+}(\tau)=\lim _{\Delta \tau \rightarrow 0, \Delta \tau>0} \frac{P\left[N^{+}(\tau, \tau+\Delta \tau)=1\right]}{\Delta \tau}$

This definition is useful only for regular processes, that is for those satisfying $\lim _{\Delta \tau \rightarrow 0, \Delta \tau>0} P\left[N^{+}(\tau, \tau+\Delta \tau)>1\right\rfloor=0$ (i.e. the probability of having more than one crossing in $[\tau, \tau+\Delta \tau]$ tends to zero when $\Delta \tau \rightarrow 0$ ). The numerator in 
Eq. (7) is nothing but the probability of having one outcrossing in $[\tau, \tau+\Delta \tau]$. Thus the outcrossing rate may be computed as follows:
- An algorithm able to find the critical design point in the $(R, Q, S, t)$ space, i.e. the instant $t^{*}$ where the (timeindependent) reliability index $\beta(t)$ is maximal as well as

$\nu^{+}(\tau)=\lim _{\Delta \tau \rightarrow 0, \Delta \tau>0} \frac{\operatorname{Prob}(\{G(\tau, \mathbf{X}(\tau, \omega))>0\} \cap\{G(\tau+\Delta \tau, \mathbf{X}(\tau+\Delta \tau, \omega)) \leq 0\})}{\Delta \tau}$

The mean number of outcrossing $E\left[N^{+}(0, t)\right]$ then reads:

$E\left[N^{+}(0, t)\right]=\int_{0}^{t} \nu^{+}(\tau) \mathrm{d} \tau$

In case of stationary problems, the outcrossing rate $\nu^{+}$is constant. Thus the latter integral simplifies into:

$E\left[N^{+}(0, t)\right]=\nu^{+} t$

\section{Computing the outcrossing rate}

\subsection{Analytical formulations and asymptotic integration}

Several analytical results regarding the outcrossing rate of a random process through a threshold are available in the literature:

- Rice's formula [18,19] for scalar differentiable processes, which has been generalized by Belayev [20] for vector differentiable processes.

- Specialization to differentiable Gaussian processes [21]

- Case of scalar and vector rectangular wave renewal processes [22].

The results mentioned above apply when a single scalar or vector process enters the problem statement. However, modelling real-life problems also requires the introduction of random variables and/or random parameters. According to the classification by Schall et al. [2], the former will be referred to as $R$ variables, which are non-ergodic in nature. The authors also introduce random ergodic sequences denoted by $Q$ and random processes $S$. The set $\mathbf{X}(t, \omega)$ is the collection of all $R, Q$, $S$ variables.

The analytical results mentioned above may be used conditioned to the values of $(R=r, Q=q)$. To get the end result, expectation with respect to these variables has to be taken [4]:

$E\left[N^{+}(0, t)\right]=E_{R, Q}\left[E\left[N^{+}(0, t \mid r, q)\right]\right]=E_{R, Q}\left[\int_{0}^{t} \nu^{+}(\tau \mid r, q) \mathrm{d} \tau\right]$

The time integration in Eq. (11) is performed using Laplace integration. The expectation operation is carried out using a FORM/SORM approach [1]. The whole procedure requires: the corresponding design point $P^{*}\left(t^{*}\right)$. The AbdoRackwitz algorithm [23] may be used.

- Analytical derivations for the Laplace integration [24,25].

This asymptotic approach is a second order method together with an approximate time integration (also to the second order).

The whole package is implemented in the software COMREL-TV $^{\circledR}$ [15], which will be used in the sequel for benchmarking purposes.

\subsection{System reliability approach: the PHI2 method}

The outcrossing rate is computed by considering the twocomponent parallel system reliability analysis in Eq. (8). It consists in making two successive analyses using Simulation or First Order Approximation Method (FORM), and evaluating the binormal cumulative distribution function as firstly presented by Hagen and Tvedt [5] and then used by $\mathrm{Li}$ and Der Kiureghian [6]. The differences with the previous applications of this approach are:

1. We consider the limit state function itself (i.e. $G(t$, $\mathbf{X}(t, \omega)))$ as the random process whose outcrossing of the zero level (limit state surface) is to characterize.

2. The $S$ random processes involved in $G(t, \mathbf{X}(t, \omega))$ do not need to be discretized, in contrast to Der Kiureghian [9].

3. We do not make a first order Taylor expansion of the limit state at $t+\Delta t$, in contrast to what was done by Hagen and Tvedt [5].

Replacing the limit passage in Eq. (8) by a finitedifference-like operation leads to the following definition:

$$
\begin{aligned}
& \nu_{\mathrm{PH} 2}^{+}(\tau) \\
& =\frac{\operatorname{Prob}(\{G(\tau, \mathbf{X}(\tau, \omega))>0\} \cap\{G(\tau+\Delta \tau, \mathbf{X}(\tau+\Delta \tau, \omega)) \leq 0\})}{\Delta \tau}
\end{aligned}
$$

In this expression, the time increment $\Delta \tau$ has to be selected properly. This will be discussed in the sequel.

Using the FORM [26], the approach summarizes as follows:

- The (time-invariant) reliability index $\beta(\tau)$ associated with the event $\{G(\tau, \mathbf{X}(\tau, \omega)) \leq 0\}$ is computed after 
having frozen $\tau$ in all functions of time and having replaced the random processes $X_{k}(t, \omega)$ by random variables $X_{k}^{(1)}(\omega)$. Classical FORM analysis corresponds to approximating the limit state surface by the hyperplane $\alpha(\tau) \cdot \mathbf{u}+\beta(\tau)=0$ in the standard normal space. As a consequence, the reliability index associated with the event $\{G(\tau, \mathbf{X}(\tau, \omega))>0\}$ is $-\beta(\tau)$.

- The reliability index $\beta(\tau+\Delta \tau)$ associated with the event $\{G(\tau+\Delta \tau, \mathbf{X}(\tau+\Delta \tau, \omega)) \leq 0\}$ is computed by another FORM analysis. It is important to notice that the random processes $X_{k}(t, \omega)$ are now replaced by another set of random variables $X_{k}^{(2)}(\omega)$ that are different from, although correlated with the $X_{k}^{(1)}(\omega)$. The correlation coefficient writes

$\rho\left(X_{k}^{(1)}, X_{k}^{(2)}\right)=\rho_{X_{k}}(\tau, \tau+\Delta \tau)$

where $\rho_{X_{k}}\left(t_{1}, t_{2}\right)$ denotes the autocorrelation coefficient function of process $X_{k}$. The approximate limit state surface writes $\alpha(\tau+\Delta \tau) \cdot \mathbf{u}+\beta(\tau+\Delta \tau)=0$ in the standard normal space. The corresponding reliability index is $\beta(\tau+\Delta \tau)$.In practice, if $X_{k}(t, \omega)$ is a standard normal process with autocorrelation coefficient function $\rho_{X_{k}}\left(t_{1}, t_{2}\right)$, the couple $\left\{X_{k}^{(1)}, X_{k}^{(2)}\right\}$ may be uncorrelated using Cholesky decomposition of its correlation matrix

$$
\left(\begin{array}{ll}
1 & \rho_{X_{k}}(\tau, \tau+\Delta \tau) \\
\rho_{X_{k}}(\tau, \tau+\Delta \tau) & 1
\end{array}\right)
$$

and independent standard normal variables $\left(\xi_{1}, \xi_{2}\right)$ as follows:

$$
\left\{\begin{array}{l}
X_{k}^{(1)}=\xi_{1} \\
X_{k}^{(2)}=\rho_{X_{k}}(\tau, \tau+\Delta \tau) \xi_{1}+\sqrt{1-\rho_{X_{k}}^{2}(\tau, \tau+\Delta \tau)} \xi_{2}
\end{array}\right.
$$

- Denoting the correlation between the two events $\{G(\tau$, $\mathbf{X}(\tau, \omega))>0\}$ and $\{G(\tau+\Delta \tau, \mathbf{X}(\tau+\Delta \tau, \omega)) \leq 0\}$ by

$\rho_{G}(\tau, \tau+\Delta \tau)=-\boldsymbol{\alpha}(\tau) \cdot \boldsymbol{\alpha}(\tau+\Delta \tau)$

the usual expression for the probability of failure of a parallel system [27] leads to:

$\nu_{\mathrm{PH} 2}^{+}(\tau)=\frac{\Phi_{2}\left[\beta(\tau),-\beta(\tau+\Delta \tau) ; \rho_{G}(\tau, \tau+\Delta \tau)\right]}{\Delta \tau}$

In the latter equation, $\Phi_{2}$ stands for the binormal cumulative distribution function.

Fig. 1 shows the probability $\nu_{\mathrm{PHI} 2}^{+} \Delta \tau$ in FORM approximation in the space of the standard variables $\mathbf{U}=T(\mathbf{X})$ where $T$ is an isoprobabilistic transformation. In this figure, $\beta(\tau)$ and $\beta(\tau+\Delta \tau)$ are the reliability indices related to the events $\{G(\tau, \mathbf{X}(\tau, \omega)) \leq 0\}$ and $\{G(\tau+\Delta \tau, \mathbf{X}(\tau+\Delta \tau, \omega)) \leq$ $0\}$, respectively. Function $\phi_{2}$ denotes the probability density function of the binormal law. The probability $\nu_{\mathrm{PHI} 2}^{+} \Delta \tau$ is represented by the integral of $\phi_{2}$ over the hatched volume.

In contrast with the asymptotic approach, the PHI2 method is a first order method for the computation of

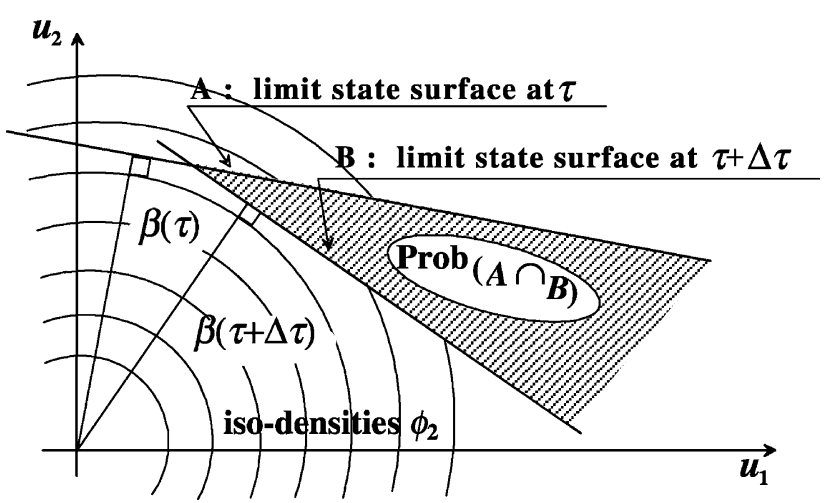

Fig. 1. Computation of $\nu_{\mathrm{PH} 2}^{+}(\tau)$ using system reliability analysis in FORM approximation.

the outcrossing rate whereas the integration over time can be made numerically exact.

Discussion on time increment. For an accurate evaluation of $\nu_{\mathrm{PHI} 2}^{+}(\tau)$, it is necessary to select the time increment $\Delta \tau$ properly. It has to be sufficiently small since it enters a finitedifference-like definition (Eq. (12)). Moreover, at most one crossing should occur during $\Delta \tau$. However, too small values would lead to numerical instabilities (among other reasons, the correlation coefficient in Eq. (15) tends to -1 ). Numerical investigations have shown that the values $\{\Delta \tau \mid$ $\left.\rho_{X_{k}}(\tau, \tau+\Delta \tau)=0.99-0.995\right\}$ provide accurate results.

\subsection{Monte-Carlo simulation}

Monte-Carlo simulation is a general method to solve reliability problems that gives accurate results if the number of samples is large enough. Typically $10^{n+2}-10^{n+3}$ samples are needed to compute accurately a probability of failure of $10^{-n}$. In case when a random process appears in the limit state function, a strategy to generate trajectories of the process has to be selected.

The usual approach is based on the random process discretization, i.e. its representation by a finite set of random variables. Several schemes are presented in Schuëller [27] and Sudret and Der Kiureghian [28].

\subsubsection{Discretization of random processes}

The method retained in the present paper is the so-called Expansion Optimal Linear Estimation method (EOLE) [29]. Let us consider a scalar Gaussian process $X(t, \omega)$ characterized by its mean value $m(t)$, standard deviation $\sigma(t)$ and autocorrelation coefficient function $\rho_{X}\left(t_{1}, t_{2}\right)$. To discretize the process, $P$ points $t_{i}, i=1, \ldots, P$ are selected within the time interval $[0, T]$ under consideration $\left(t_{1}=0, t_{P}=T\right)$. The EOLE expansion reads:

$X(t, \omega) \approx m(t)+\sigma(t) \sum_{i=1}^{r} \frac{\xi_{i}(\omega)}{\sqrt{\lambda_{i}}} \phi_{i}^{\mathrm{T}} C_{t, t_{i}}(t)$

In this expression, the following notation is used:

- $\xi_{i}(\omega)$ are independent standard normal random variables, 
- $\left(\lambda_{i}, \phi_{i}\right)$ are the eigenvalues and eigenvectors of the correlation matrix $\mathbf{C}$, whose generic term is $C_{i j}=$ $\left\{\rho_{X}\left(t_{i}, t_{j}\right), i, j=1, \ldots, P\right\}$ (note that the eigenvalues are sorted in decreasing order),

- $C_{t, t_{i}}(t)$ is a time-dependent vector, whose components are $C_{t, t_{i}}(t)=\rho_{X}\left(t, t_{i}\right), i=1, \ldots, P$,

- $r \leq P$ is the number of terms in the summation, corresponding to the $r$ largest eigenvalues of $\mathbf{C}$.

It is observed that this expansion is a kind of 'variables splitting' since the process is replaced by a sum of products of random variables by functions of time. The EOLE expansion is computed by a Matlab routine as presented in Sudret and Der Kiureghian [28], which can be downloaded at http://www.ce.berkeley.edu/ haukaas under the item FERUM. An error estimator, which allows to evaluate the accuracy of the discretization is given by:

$\operatorname{err}(t)=1-\sum_{i=1}^{r} \frac{1}{\lambda_{i}}\left(\phi_{i}^{\mathrm{T}} C_{t, t_{i}}(t)\right)^{2}$

\subsubsection{Computation of the outcrossing rate and failure probability}

Suppose a trajectory of the limit state function $G(t$, $\left.\mathbf{X}\left(t, \omega_{0}\right)\right) \leq 0$ is simulated in the time interval $[0, t]$. Its values are stored in an array $\left\{G\left(t_{i}\right), t_{i}=(i-1) \cdot \Delta t, \times\right.$ $i=1, \ldots, N\}$ where $N$ is the number of points used in the discretization and $\Delta t=t /(N-1)$ is the sampling step. $N$ can be different from $P$. Using Monte-Carlo simulation, $N_{\mathrm{MC}}$ such trajectories are considered. Let $k_{i}, i=1, \ldots, N-1$ be a set of counters that are incremented by one each time the current trajectory presents an outcrossing of the limit state surface within the interval $\left[t_{i}, t_{i+1}\right]$. The Monte-Carlo estimate of the outcrossing rate $\tilde{\nu}_{\mathrm{MC}}^{+}(t)$ then is:

$\tilde{\nu}_{\mathrm{MC}}^{+}\left(t_{i}\right)=\frac{k_{i}}{N_{\mathrm{MC}} \cdot \Delta t}$

The variance of this estimator is:

$\operatorname{Var}\left[\tilde{\nu}_{\mathrm{MC}}^{+}\left(t_{i}\right)\right]=\frac{1}{\left(N_{\mathrm{MC}}-1\right) \Delta t^{2}} \frac{k_{i}}{N_{\mathrm{MC}}}\left(1-\frac{k_{i}}{N_{\mathrm{MC}}}\right)$

The estimated upper bound on the probability of failure $\tilde{P}_{f, c \mathrm{MC}}\left(0, t_{i}\right)$ on any time interval $\left[0, t_{i}\right]$ (Eq. (6)) is given by integrating Eq. (19) over $\left[0, t_{i}\right]$, which yields:

$\tilde{P}_{f, c \mathrm{MC}}\left(0, t_{i}\right)=\frac{1}{N_{\mathrm{MC}}} \sum_{j=1}^{i-1} k_{j}, \quad 2 \leq i \leq N$

The variance of this estimator is:

$\operatorname{Var}\left(\tilde{P}_{f, c \mathrm{MC}}\left(0, t_{i}\right)\right)=\frac{1}{N_{\mathrm{MC}}-1}\left[\frac{1}{N_{\mathrm{MC}}} \sum_{j=1}^{i-1} k_{j}-\left(\frac{1}{N_{\mathrm{MC}}} \sum_{j=1}^{i-1} k_{j}\right)^{2}\right]$
Eqs. (19)-(22) allow to evaluate the coefficient of variation of the various simulations namely

$V\left[\tilde{\nu}_{\mathrm{MC}}^{+}\left(t_{i}\right)\right]=\frac{\sqrt{\operatorname{Var}\left(\tilde{\nu}_{\mathrm{MC}}^{+}\left(t_{i}\right)\right)}}{\tilde{\nu}_{\mathrm{MC}}^{+}\left(t_{i}\right)}$ and
$V\left[\tilde{P}_{f, c \mathrm{MC}}\left(0, t_{i}\right)\right]=\frac{\sqrt{\operatorname{Var}\left(\tilde{P}_{f, c \mathrm{MC}}\left(0, t_{i}\right)\right)}}{\tilde{P}_{f, c \mathrm{MC}}\left(0, t_{i}\right)}$

In practice, for each simulated trajectory, the condition $\left\{\left\{G\left(t_{i}, \mathbf{X}\left(t_{i}, \omega_{0}\right)\right)>0\right\} \cap\left\{G\left(t_{i+1}, \mathbf{X}\left(t_{i+1}, \omega_{0}\right)\right) \leq 0\right\}\right\}$ is checked at all time instants $t_{1}, \ldots, t_{N-1}$, and if it holds, the corresponding value of $k_{i}$ is incremented. This allows to compute the total number of outcrossings corresponding to the $N_{\mathrm{MC}}$ trajectories, i.e. possibly several outcrossings for each trajectory.

If one is interested in the probability of failure associated with the first outcrossing (i.e. the cumulative failure probability itself and not the upper bound of it), the counting method is slightly modified: once an outcrossing has been detected in the current trajectory, the remaining part of it is not checked and the program gets to the next trajectory.

\section{Benchmark of the approaches}

In this section, three examples are dealt with using various approaches:

- The AsM, as implemented in COMREL-TV V.7.1 [15]. It allows to compute the upper bound of the failure probability using an asymptotic integration.

- The PHI2 method. COMREL-TI V.7.1 is used to carry out the required time-invariant FORM analyses. Note that the default parameters for FORM analysis are used, except the tolerance on the convergence, which is set equal to $10^{-3}$. It allows to compute the upper bound of the failure probability using an 'exact' (i.e. only numerical) integration.

- Monte-Carlo simulation. Matlab routines are used to discretize the random process and perform the simulation.

Simple examples with linear limit state involving Gaussian random variables and a scalar Gaussian process have been studied in Andrieu-Renaud [13], Sudret et al. [14] and Andrieu et al. [30]. They show that PHI2 and AsM give results with a similar trend and that $\mathrm{PHI} 2$ is more accurate than AsM (with respect to available analytical solutions) in such cases. The three examples presented in this paper involve non-linear limit states, a scalar process in the first example and a vector process in the two others (Gaussian in the first case and lognormal in the second case).

\subsection{First example: corroded beam under random loading}

The first example deals with a steel bending beam. Its length is $L=5 \mathrm{~m}$, its cross-section is rectangular 

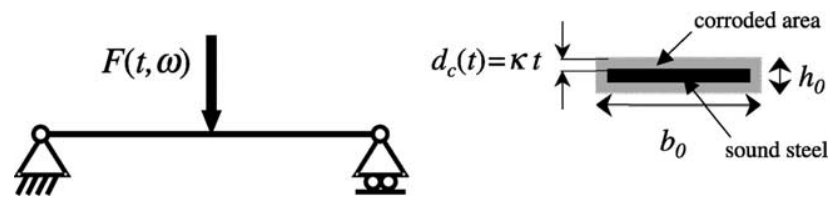

Fig. 2. Corroded bending beam submitted to dead load and random loading.

$\left(b_{0}=0.2 \mathrm{~m}, h_{0}=0.04 \mathrm{~m}\right)$. This beam is submitted to dead load (denoting by $\rho_{s t}=78.5 \mathrm{kN} / \mathrm{m}^{3}$ the steel force density, this load is equal to $\left.p=\rho_{s t} b_{0} h_{0}(\mathrm{~N} / \mathrm{m})\right)$ as well as a pinpoint load $F$ applied at mid span (Fig. 2).

Let us denote by $f_{y}$ the steel yield stress. It is now supposed that the steel beam corrodes in time: the corrosion phenomenon is supposed to start at $t=0$, is isotropic all around the beam cross-section and evolves linearly in time. Assuming that the corroded areas have lost all mechanical stiffness, the sound cross-section at time $t$ writes (Fig. 2)

$s(t)=b(t) \cdot h(t)$ where $b(t)=b_{0}-2 \kappa t ; h(t)=h_{0}-2 \kappa t$

where parameter $\kappa=0.03 \mathrm{~mm} /$ year controls the corrosion kinetics. The bending moment at midspan associated with dead- and pinpoint loads reads:

$M(t)=\frac{F(t) L}{4}+\frac{\rho_{s t} b_{0} h_{0} L^{2}}{8}$

The ultimate bending moment corresponding to the appearing of a plastic hinge in the section reads:

$M_{u}(t)=\frac{b(t) h^{2}(t)}{4} f_{y}$

Thus the limit state function associated with the failure of the beam reads

$$
\begin{aligned}
G(t, \mathbf{X}(t, \omega))= & \frac{b(t, \omega) h^{2}(t, \omega) f_{y}(\omega)}{4} \\
& -\left(\frac{F(t, \omega) L}{4}+\frac{\rho_{s t} b_{0}(\omega) h_{0}(\omega) L^{2}}{8}\right)
\end{aligned}
$$

where the dependency of the dimensions to time have been specified in Eq. (23). The random input parameters are gathered in Table 1 . The time interval under consideration is [0, 20 years].

Table 1

\begin{tabular}{|c|c|c|c|c|}
\hline Parameter & $\begin{array}{l}\text { Type of } \\
\text { distribution }\end{array}$ & Mean & $\begin{array}{l}\text { Coefficient of } \\
\text { variation }(\%)\end{array}$ & $\begin{array}{l}\text { Autocorrelation } \\
\text { coefficient } \\
\text { function }\end{array}$ \\
\hline $\begin{array}{l}\text { Steel yield } \\
\text { stress } f_{y}\end{array}$ & Lognormal & $240 \mathrm{MPa}$ & 10 & NA \\
\hline $\begin{array}{l}\text { Beam breadth } \\
b_{0}\end{array}$ & Lognormal & $0.2 \mathrm{~m}$ & 5 & NA \\
\hline $\begin{array}{l}\text { Beam height } \\
h_{0}\end{array}$ & Lognormal & $0.04 \mathrm{~m}$ & 10 & NA \\
\hline Load $F$ & Gaussian & $3500 \mathrm{~N}$ & 20 & Eq. (27) \\
\hline
\end{tabular}

Corroded bending steel beam-random variables and parameters
The load is modelled as a stationary Gaussian random process (Table 1). The autocorrelation coefficient function is of exponential square type (Eq. (27)), with a correlation length $\lambda=1$ month (i.e. $8.33 \times 10^{-2}$ year)

$\rho_{F}\left(t_{1}, t_{2}\right)=\exp \left(-\left(\frac{t_{2}-t_{1}}{\lambda}\right)^{2}\right)$

It is emphasized that the time scale corresponding to the loading and that corresponding to the corrosion are completely different, without introducing any difficulty in the PHI2 solving strategy.

The initial probability of failure is computed by a timeinvariant FORM analysis. It yields $\beta_{0}=4.536$, i.e. $P_{f, i}(0)=2.87 \times 10^{-6}$. The evolution in time of the upper bound to the probability of failure is represented in Fig. 3.

As far as Monte-Carlo simulation is concerned, the EOLE decomposition was based on 66 points on 20 oneyear-long intervals (i.e. on [0, 1 year] then [1 year, 2 years]...until [19 years, 20 years]), from which $r=15$ terms were retained in each time interval (Eq. (17)). This corresponds to a discretization error (Eq. (18)) of $1 \%$. To compute the outcrossing rate at $t=1$ year with $\Delta t=0.2$ month, 1,000,000 trajectories were simulated, then $t=2$ years with the same value for $\Delta t$ until $t=20$ years. Then the outcrossing rate is numerically integrated to obtain the failure probability. The final coefficient of variation $V$ of the simulation is given in Table 2.

The upper bound to the probability of failure, given by simulation, AsM and PHI2 methods is depicted in Fig. 3. The value corresponding to the lower bound of $P_{f, c}$ is given as well. All approaches give a similar trend. PHI 2 provides results that are closer to those obtained by Monte-Carlo simulation than AsM. However, the discrepancies between PHI2 and AsM may be a1cceptable from an engineering point of view.

It is now interesting to compare the number of calls to the limit state function required by PHI2 and AsM. A parametric study giving the point-in-time reliability index $\beta(t)$, using FORM within COMREL-TI V.7.1, for $t=$ $1, \ldots, 20$ years requires 787 calls. To compute the outcrossing rate, PHI2 requires two successive FORM analyses, i.e. 1774 calls. If a complete parametric study giving $P_{f, c}(0, t)$ for $t=1, \ldots, 20$ years is carried out using COMREL-TV V.7.1, 5179 calls are needed. In this case, $\mathrm{PHI} 2$ is computationally less expensive.

\subsection{Second example: use of vector processes}

The limit state function under consideration is chosen to take into account two random variables and a vector random process:

$$
\begin{aligned}
G(t, \mathbf{X}(t, \omega))= & R_{1}(\omega)+R_{2}(\omega)-0.5 \sqrt{2 t}-\left(0.2 S_{1}(t, \omega)\right. \\
& \left.+0.3 S_{2}(t, \omega)\right)^{2}
\end{aligned}
$$



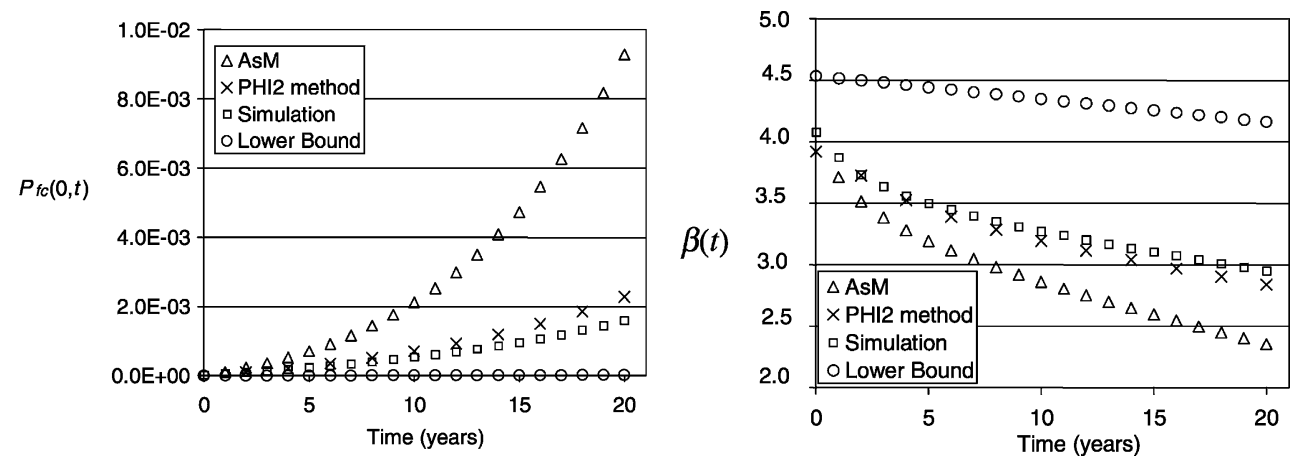

Fig. 3. Corroded bending steel beam—probability of failure and reliability index vs. time.

Table 2

Corroded bending beam: probability of failure over [0, 20 years $]$

\begin{tabular}{ll}
\hline Method & $P_{f, c}$ \\
\hline AsM & $9.29 \times 10^{-3}$ \\
PHI2 & $2.28 \times 10^{-3}$ \\
MC simulation $(V)$ & $1.59 \times 10^{-3}(2.5 \%)$ \\
Lower bound & $1.59 \times 10^{-5}$ \\
\hline
\end{tabular}

It includes two random variables $R_{1}$ and $R_{2}$, and a vector random process $\mathbf{S}$ with two components $S_{1}$ and $S_{2}$ whose correlation coefficient functions are defined by

$\rho_{S_{1} S_{1}}\left(t_{1}, t_{2}\right)=\exp \left(-\left(\frac{t_{2}-t_{1}}{\lambda_{1}}\right)^{2}\right)$

$\rho_{S_{2} S_{2}}\left(t_{1}, t_{2}\right)=\exp \left(-\left(\frac{t_{2}-t_{1}}{\lambda_{2}}\right)^{2}\right)$

$\rho_{S_{1} S_{2}}\left(t_{1}, t_{2}\right)=0.3 \exp \left(-\left(\frac{t_{2}-t_{1}}{\lambda_{3}}\right)^{2}\right)$

where $\lambda_{1}=1, \lambda_{2}=\sqrt{2} / 2$ and $\lambda_{3}=1$.

Two cases are considered: in the first case the process is supposed to be Gaussian, and it is lognormal in the second case. Note that the correlation coefficient functions are given by Eq. (28) in both cases.

The random input parameters are gathered in Table 3. The time interval under consideration is $[0,16]$.

All components of the random vector process should be replaced by corresponding (correlated) random variables in the time-independent FORM analyses carried out at $t$ and $t+\Delta t$. The rest of the approach remains unchanged. Nataf

Table 3

Second example: random variables and parameters

\begin{tabular}{llcll}
\hline Parameter & $\begin{array}{l}\text { Type of } \\
\text { distribution }\end{array}$ & Mean & $\begin{array}{l}\text { Standard } \\
\text { deviation }\end{array}$ & $\begin{array}{l}\text { Auto and } \\
\text { cross-correlation } \\
\text { coefficient function }\end{array}$ \\
\hline$R_{1}$ & Exponential & 7 & 3 & NA \\
$R_{2}$ & Lognormal & 25 & 7 & NA \\
$S_{1}$ & Gaussian/lognormal & 2.8 & 1.4 & Eq. (28) \\
$S_{2}$ & Gaussian/lognormal & 7 & 1 & \\
\hline
\end{tabular}

transformation can be dealt with without additional implementation. All the results are presented for both cases in Table 4, Figs. 4 and 5.

In this case, both approaches give a similar trend in the evolution in time of the reliability index. AsM provides higher probabilities of failure than PHI2. However, no reference results are available in this case.

As in the previous example, it is now interesting to compare the number of calls to the limit state function required by PHI 2 and AsM. To compute the outcrossing rate, PHI2 requires 547 calls for the Gaussian case (resp. 590 in the lognormal case). If a complete parametric study giving $P_{f, c}(0, t)$ for $t=1, \ldots, 16$ years is carried out using COMREL-TV V.7.1, 2195 calls would be needed for the Gaussian case (resp. 3540 in the lognormal case). In this case, PHI2 would be again computationally less expensive.

\section{Durability of a beam submitted to creep and pinpoint load}

\subsection{Problem statement}

The structure under consideration is a concrete beam subjected to creep and to a pinpoint load. The beam length is $L=10 \mathrm{~m}$, its cross-section is rectangular $(b=0.2 \mathrm{~m}$, $h=0.2 \mathrm{~m})$. The concrete compression strength is $f_{c, 28}=30 \mathrm{MPa}$. The concrete instantaneous Young's modulus is $E_{28}=11,000\left(f_{c, 28}\right)^{1 / 3}=34,180 \mathrm{MPa}$.

This beam is subjected to dead load (denoting by $\rho=2500 \mathrm{~kg} / \mathrm{m}^{3}$ the concrete mass density, this load is equal to $p=\rho b h=1000 \mathrm{~N} / \mathrm{m}$ ) as well as a pinpoint load $S$ applied at midspan.

Table 4

Second example (vector case): probability of failure over $[0,16]$

\begin{tabular}{lll}
\hline Method & $P_{f, c} \mathbf{S}$ Gaussian & $P_{f, c} \mathbf{S}$ lognormal \\
\hline AsM & $7.08 \times 10^{-3}$ & $2.81 \times 10^{-2}$ \\
PHI2 & $5.04 \times 10^{-3}$ & $2.04 \times 10^{-2}$ \\
Lower bound & $0.24 \times 10^{-3}$ & $0.09 \times 10^{-2}$
\end{tabular}



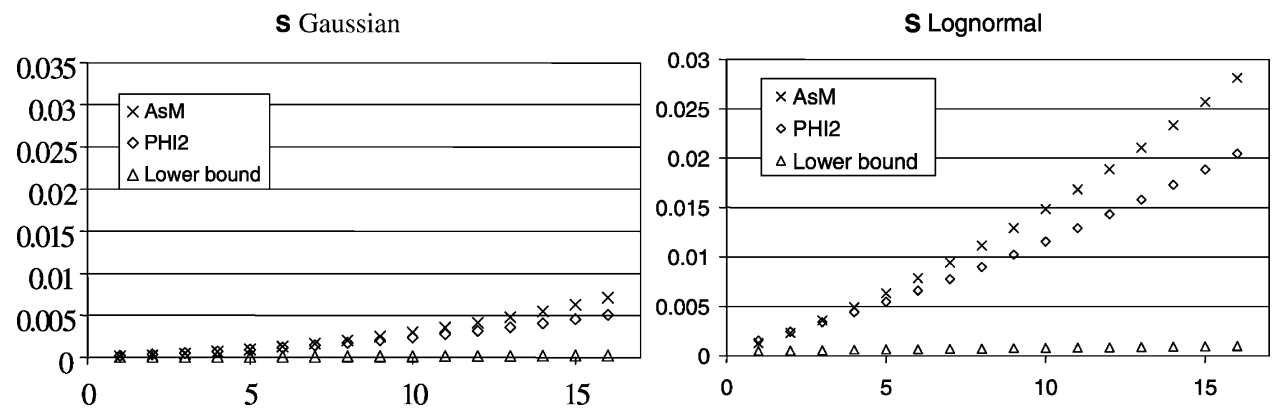

Fig. 4. Vectorial case-probability of failure vs. time.
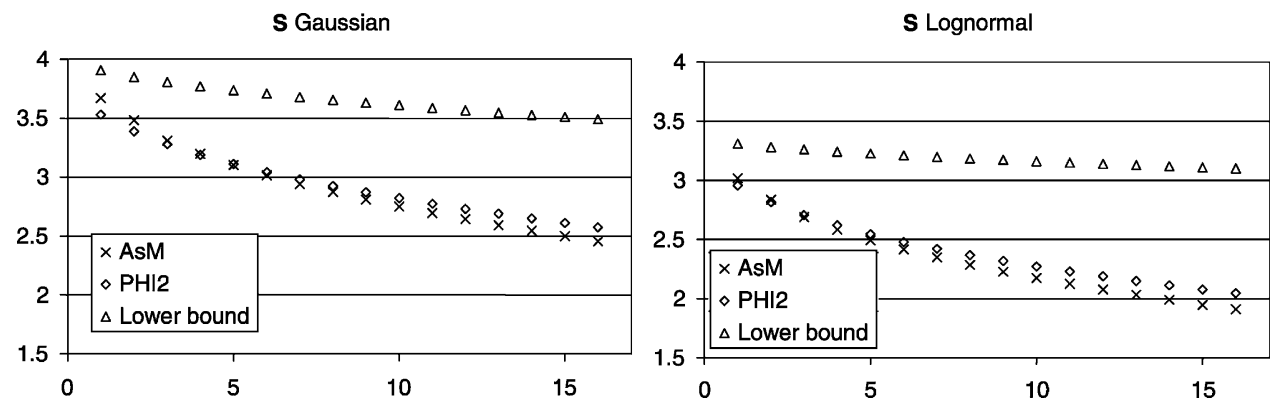

Fig. 5. Vectorial case-index reliability vs. time.

The instantaneous maximum deflection at this point due to the dead load is

$\delta_{w}^{0}=\frac{5}{384} \frac{p L^{4}}{E_{28} I}$

where $I=b h^{3} / 12$ is the beam inertia.

Let us note $J\left(t, t_{c}\right)$, the uniaxial compliance of concrete which depends both on time $t$ and time $t_{c}$ corresponding to the loading of the structure. The uniaxial strain corresponding to axial stress $\sigma$ reads:

$\varepsilon(t)=J\left(t, t_{c}\right) \sigma$

The French codified model for creep is cast as [31]

$J\left(t, t_{c}\right)=\frac{1}{E_{28}}\left(1+\varphi\left(t, t_{c}\right)\right)$

where $\varphi\left(t, t_{c}\right)$ is:

$\varphi\left(t, t_{c}\right)=\varphi_{\infty} \frac{\sqrt{t-t_{c}}}{\sqrt{t-t_{c}}+K}$

In this study, the model parameters are computed or selected as follows:

- $\varphi_{\infty}$ can be calculated as a function of the concrete formulation, the relative humidity of the environment and the dimensions of the beam cross-section. It is set equal to 2 in the sequel.

- $t_{c}=28$ days,

- $K=15.8$ (computed also from the dimensions of the beam cross-section).

When creep is taken into account, the deflection under dead load is:

$$
\delta_{W}(t)=\delta_{W}^{0}\left(1+\varphi_{\infty} \frac{\sqrt{t-t_{c}}}{\sqrt{t-t_{c}}+K}\right)
$$

The live load is a pinpoint load $S$ applied at midspan during $\left[t_{d}, t_{f}\right]$ where $t_{f}=t_{d}+1$ year. This duration is introduced by means of the Heaviside function $H$. The resulting deflection at this point, if the duration is sufficiently short so that the instantaneous Young's modulus may be used, is:

$\delta_{S}(t)=\frac{S L^{3}}{48 E_{28} I}\left[H\left(t-t_{d}\right)-H\left(t-t_{f}\right)\right]$

Modelling the live load this way allows us representing a mechanical system placed in an environment that can present exceptional configurations.

\subsection{Reliability problem statement}

The failure criterion is defined by the exceeding of a limit deflection $\delta_{\text {lim }}=10 \mathrm{~cm}$. The limit state function associated with the failure of the beam reads:

$G(t, \mathbf{X}(t, \omega))=\delta_{\lim }-\delta_{w}(t)-\delta_{S}(t)$

The random input parameters are presented in Table 5. The study is carried out over [0,50 years].

Three cases are studied:

1. $S$ is a random variable applied onto the beam middle point at $t_{1}=10,20, \ldots, 50$ years during one year, $t_{f}=$ $t_{d}+1$ year.

2. $S$ is a stochastic process applied onto the beam middle point at $t_{1}=10,20, \ldots, 50$ years. Its coefficient 
Table 5

Concrete beam under creep and live load: random variables

\begin{tabular}{lllll}
\hline Parameter & $\begin{array}{l}\text { Type of } \\
\text { distribution }\end{array}$ & Mean & Cov (\%) & $\begin{array}{l}\text { Autocorrelation } \\
\text { coefficient function }\end{array}$ \\
\hline$P$ & Lognormal & $1000 \mathrm{~N} / \mathrm{m}$ & 10 & NA \\
$\varphi_{\infty}$ & Lognormal & 2 & 20 & NA \\
$K$ & Lognormal & 15.8 & 30 & NA \\
$S$ & Gauss & $5000 \mathrm{~N}$ & 20 & Case (1): NA \\
& & & & Case (2): Eq. (36) \\
& & & & Case (3): Eq. (36) \\
\hline
\end{tabular}

correlation function is of exponential square type (Eq. (36)), with a correlation length $l=18$ days

$\rho_{S S}\left(t_{1}, t_{2}\right)=\exp \left(-\left(\frac{t_{2}-t_{1}}{l}\right)^{2}\right)$

3. $S$ is a stochastic process applied at $t_{d}$ during one year and $t_{d}$ is a random variable uniformly distributed over $[0,49$ years].

\subsection{Numerical results}

\subsubsection{First case: $S$ random variable}

In this study, time is dealt with as a parameter. Note that four calculations (i.e. four different curves corresponding to $t_{d}=$ 10, 20, 30, 40 years, respectively) are represented in Fig. 6. COMREL-TI is used to perform the FORM analysis. We can notice that the obtained point-in-time failure probability crucially increases when the pinpoint load is applied (kind of step function around this date) and decreases thereafter. This study shows that for a prescribed reliability level, e.g. $1 \%$, the structure has to be repaired before 20 years when foreseeing an exceptional event such as the pinpoint load. Nevertheless, the drawback of this first approach is that the system is considered without memory, since the computed probability of failure becomes very small after the exceptional event.

\subsubsection{Second case: $S$ stochastic process applied at $t_{d}$, where} $t_{d}$ is deterministic

The procedure in this case reads as follows:

1. Compute the reliability indices $\beta(t)$ and $\beta(t+\Delta t)$ at the different dates $t$ and $t+\Delta t$.

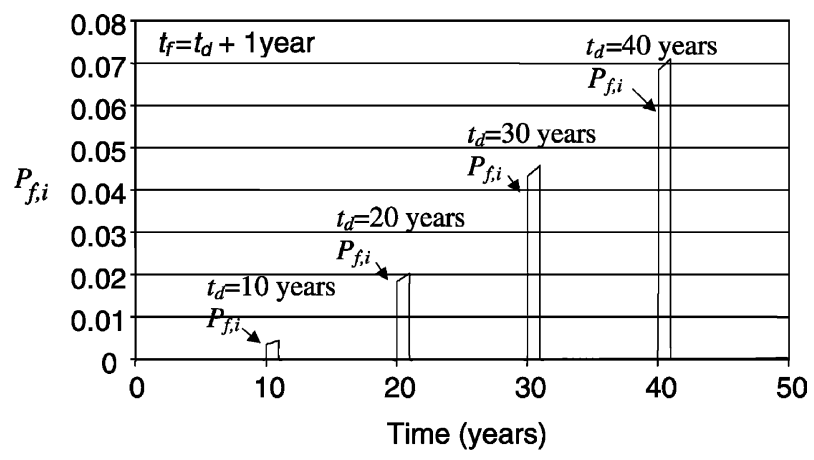

Fig. 6. Concrete beam under creep and dead load—first case: $S$ random variable-point-in-time failure probability for various values of $t_{d}$.
2. Evaluate the correlation coefficient function $\rho_{G}(t, t+\Delta t)$ of the limit state at different dates.

3. Compute the outcrossing rate and then the cumulative failure probability.

The computation of these various quantities is carried out by COMREL-TI according to the procedure explained in Section 3.2. Routines in $\mathrm{C}++$ and $\mathrm{MathCad}^{\circledR}$ have been implemented to facilitate the work.

Results are presented in Fig. 7. They show that a timevariant reliability study allows to take into account the effect of the point-in-time load. Indeed, we can see a major increase of the failure probability value when the pinpoint load is applied. Then, the probability increases slowly.

The results show that carrying out a time-variant analysis allows to consider the system as having memory, which seems more realistic than in the first case (i.e. load modelled as a random variable). On the other hand, the assumption of a prescribed $t_{d}$ means that the date at which the exceptional event happens is known, which is conflicting with the very definition of such an event.

5.3.3. Third case: $S$ stochastic process applied at $t_{d}$, where $t_{d}$ is random and uniformly distributed

For practical computation, the limit state function is rewritten as:

$G(t, \mathbf{X}(t, \omega))= \begin{cases}\delta_{\lim }-\delta_{w}(t) & \text { for } t<t_{d} \text { or } t>t_{f} \\ \delta_{\lim }-\delta_{w}(t)-\delta_{S}(t) & \text { for } t_{d} \leq t \leq t_{f}\end{cases}$

The FORM method could not be applied for computing the point-in-time reliability index $\beta(t)$ with such a formulation. Indeed, during the search of the design point, one or the other expression of Eq. (37) is used depending on the current realization of $t_{d}$, meaning a switch between two different states. Moreover, the gradient $\partial G / \partial t_{d}$ is required and is always equal to zero (precisely it would be a Dirac function which cannot be handled properly). A Monte-Carlo simulation strategy has eventually been adopted to get the quantities necessary for applying the PHI2 method.

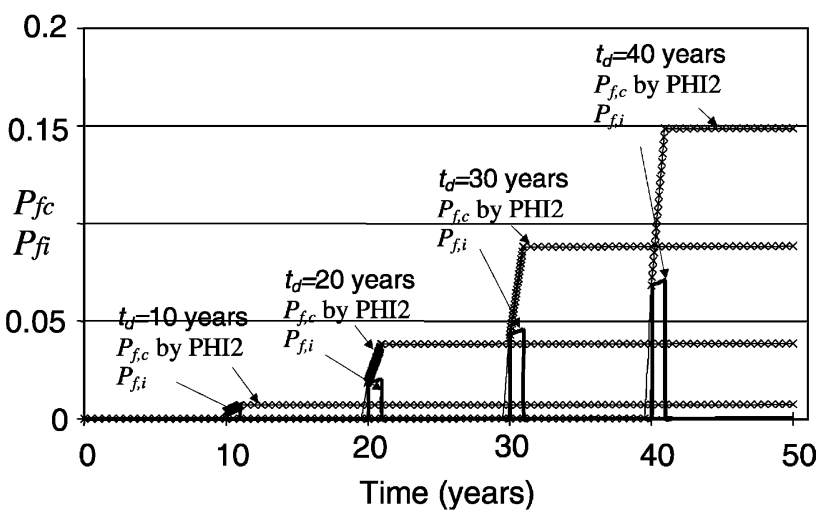

Fig. 7. Concrete beam under creep and dead load-second case: $S$ stochastic process and $t_{d}$ deterministic-failure probability for various values of $t_{d}$. 


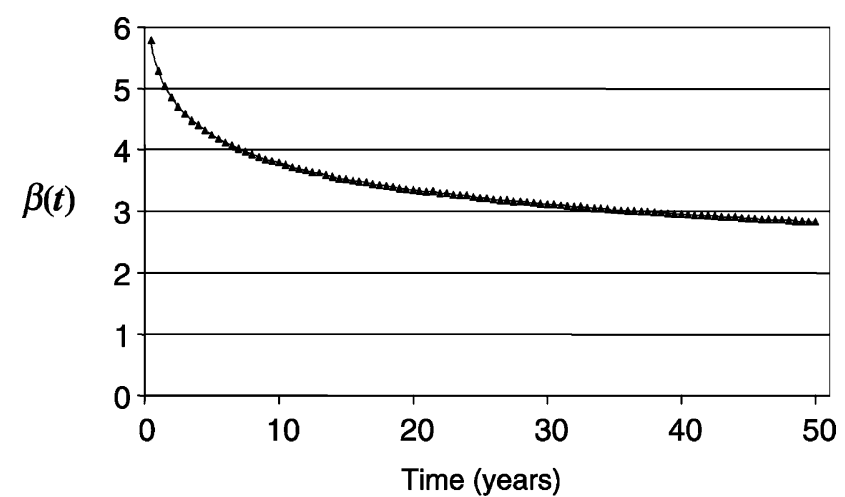

Fig. 8. Concrete beam under creep and dead load-third case: $S$ stochastic process and $t_{d}$ random variable-reliability index vs. time by simulation method.

5.3.3.1. Computation of the reliability indices $\beta(t)$ and $\beta(t+\Delta t)$. The reliability indices vs. time have been obtained by simulation. At each time step $t, 1,000,000$ samples have been used. This high number is required to get a coefficient of variation less than $5 \%$. This number can be decreased in using other methods of simulation, which has not been done in this case. The process was discretized using appropriate variables to compute the reliability indices as $\beta(t)=-\Phi^{-1}\left(P_{f, i}(t)\right)$.

The reliability index (resp. the coefficient of variation of the simulation) is plotted as a function of time in Fig. 8 (resp. Fig. 9).

5.3.3.2. Introduction of the process correlation $\rho_{S}(t, t+\Delta t)$ in the expression of the limit state at $t \Delta t$. By freezing time, the limit state at $t$ is only a function of random variables. When doing so at time $t+\Delta t$, the limit state is only function of random variables as well. However, the random variable representing the process at $t+\Delta t$ is correlated with the random variable representing the process at $t$.

Let us introduce the following notation:

- $S^{(1)}$ the random variable representing the process at $t$,

- $S^{(2)}$ the random variable representing the process at $t+$ $\Delta t$, correlated with $S^{(1)}$.

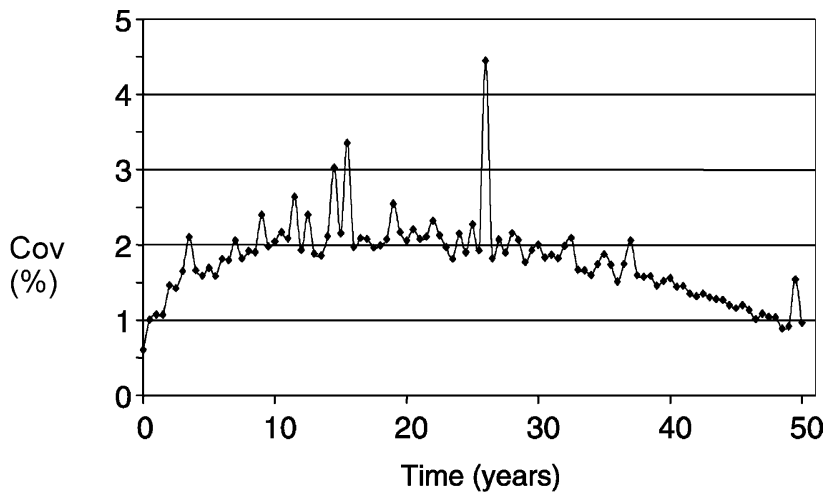

Fig. 9. Concrete beam under creep and dead load—third case: $S$ stochastic process and $t_{d}$ random variable - covariance of the simulation.
- $S^{(3)}$ the random variable representing the process at $t+$ $\Delta t$, not correlated with $S^{(1)}$,

With this notation $S^{(2)}$ may be written as a linear combination of $S^{(1)}$ and $S^{(3)}$

$S^{(2)}=\rho_{S}(t, t+\Delta t) S^{(1)}+\sqrt{1-\rho_{S}^{2}(t, t+\Delta t)} S^{(3)}$

5.3.3.3. Computation of the correlation $\rho_{G}(t, t+\Delta t)$ for the limit state at $t$ and at $t+\Delta t$. As FORM cannot be used to compute the $\alpha$-values, the computation of the correlation $\rho_{G}(t, t+\Delta t)$ used in Eq. (16) is done by simulation.

The Pearson relationship is used. It allows to compute the correlation between the elements of two $n$ dimensional sample sets $\mathbf{A}=\left\{A_{i}\right\}$ and $\mathbf{B}=\left\{B_{i}\right\}$. The empirical mean, variance and covariance of $\mathbf{A}$ and $\mathbf{B}$ may be computed first by:

$\tilde{m}_{\mathrm{A}}=\frac{1}{n} \sum_{i=0}^{n-1} A_{i}$ and $\tilde{m}_{\mathrm{B}}=\frac{1}{n} \sum_{i=0}^{n-1} B_{i}$

$\operatorname{Varr}(\mathbf{A})=\frac{1}{n-1} \sum_{i=0}^{n-1}\left(A_{i}-\tilde{m}_{\mathrm{A}}\right)^{2}$ and

$\operatorname{Vãr}(\mathbf{B})=\frac{1}{n-1} \sum_{i=0}^{n-1}\left(B_{i}-\tilde{m}_{\mathrm{B}}\right)^{2}$

$\operatorname{Covv}(\mathbf{A}, \mathbf{B})=\frac{1}{n-1} \sum_{i=0}^{n-1}\left(A_{i}-\tilde{m}_{\mathrm{A}}\right)\left(B_{i}-\tilde{m}_{\mathrm{B}}\right)$

Let $\mathbf{A}$ be the set of the realizations of $G\left(t, \mathbf{X}\left(t, \omega_{\iota}\right)\right)$ and $\mathbf{B}$ the vector of the realizations of $G\left(t+\Delta t, \mathbf{X}\left(t+\Delta t, \omega_{\iota}\right)\right)$, the correlation $\rho_{G}(t, t+\Delta t)$ is computed by:

$\rho_{G}(t, t+\Delta t)=\frac{\operatorname{Cõv}(\mathbf{A}, \mathbf{B})}{\sqrt{\operatorname{Vãr}(\mathbf{A})} \sqrt{\operatorname{Vãr}(\mathbf{B})}}$

This method is valid if the points associated with sample sets $\mathbf{A}$ and $\mathbf{B}$ fit on a straight line. This is checked and confirmed by plotting the sets (Figs. $10(t=10$ years) and $11(t=50$ years $))$.

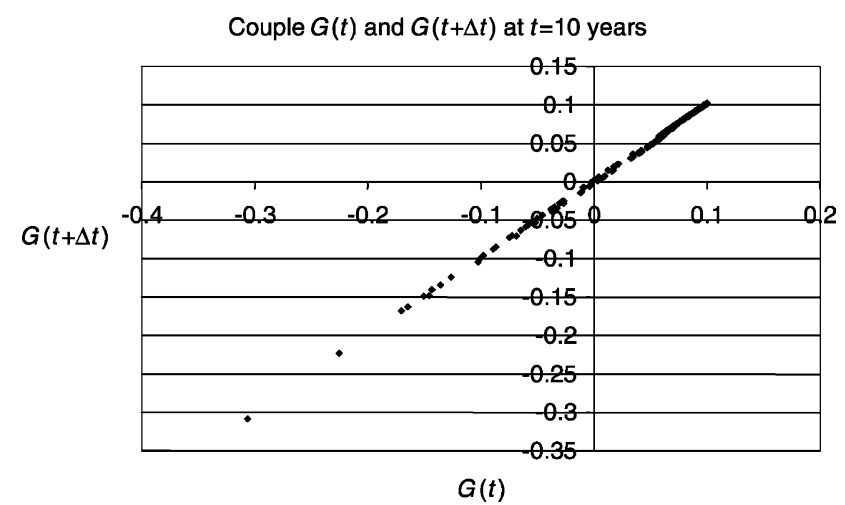

Fig. 10. Concrete beam under creep and dead load-third case: $S$ stochastic process and $t_{d}$ random variable - correlation at $t=10$ years. 


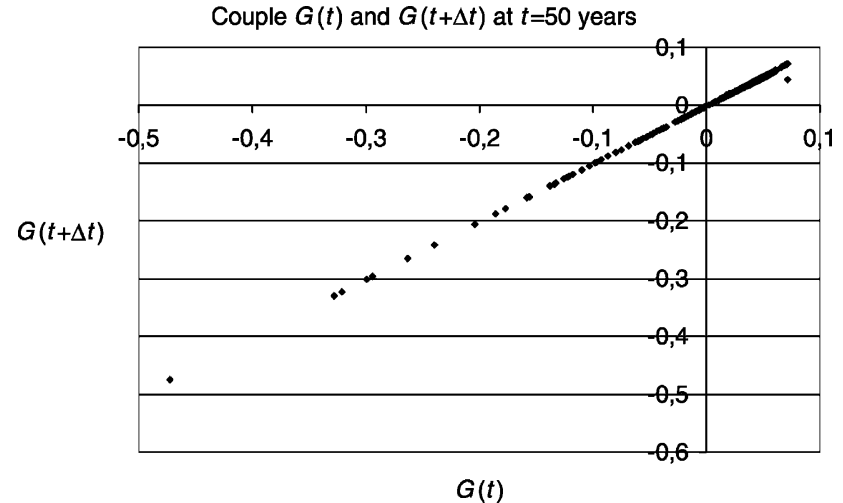

Fig. 11. Concrete beam under creep and dead load — third case: $S$ stochastic process and $t_{d}$ random variable-correlation at $t=50$ years.

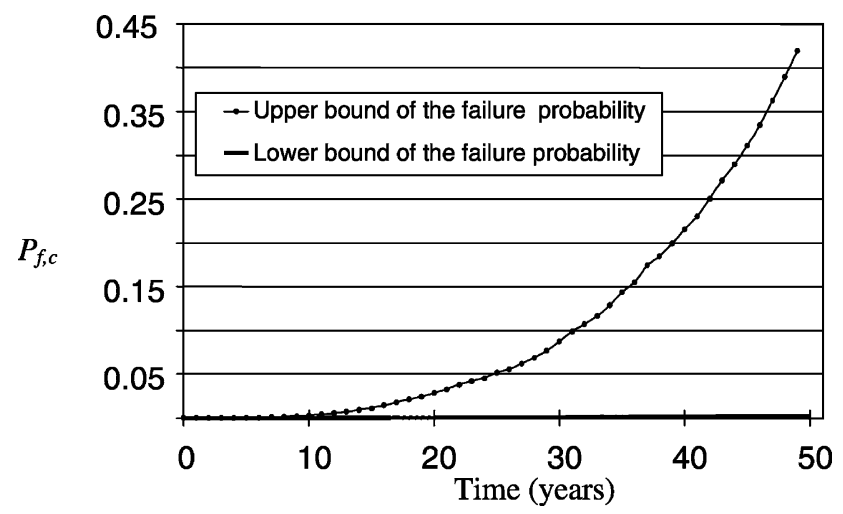

Fig. 12. Concrete beam under creep and dead load—-third case: $S$ stochastic process and $t_{d}$ random variable-cumulative failure probability.

\subsubsection{Computation of the outcrossing rate by the PHI2} method and the cumulative failure probability. Once the point-in-time reliability indices $\beta(t)$ and $\beta(t+\Delta t)$ and the correlation $\rho_{G}(t, t+\Delta t)$ have been computed as explained above, it is possible to apply Eq. (16) to get the outcrossing rate. It is then integrated in time by means of Simpson's rule. All the results are presented in Fig. 12.

We can notice that the evolution of the failure probability is regular and that it is always higher than in the previous case (i.e. when the date $t_{d}$ is not random).

The obtained results show that the modelling of the load and precisely the way it is applied highly influence the computed reliability index.

\section{Conclusion}

This paper has presented the PHI2 method for solving time-variant reliability problems. The approach specifically deals with computing the outcrossing rate appearing in timevariant reliability problems by using a classical timeinvariant parallel system analysis.

The main advantage of the PHI2 method is that it only requires time-invariant reliability tools, e.g. simulation or FORM method. As a consequence, any commercial software that contains simulation and/or FORM can be used without additional implementation.

The PHI2 method can deal with non-linear limit state including scalar or vector processes with no additional difficulty compared to the Gaussian scalar case. In all examples presented in this paper, the PHI 2 method provides results that are accurate when the comparison with a reference solution is possible.

The PHI2 method is finally illustrated on the example of a concrete beam submitted to creep and a pinpoint random load. This quite realistic problem shows the versatility of approach. Note that this problem may be solved by introducing intermittent processes in the definition of the reliability problem.

In the present paper, FORM has been used to compute the point-in-time reliability indices $\beta(t)$ required by the PHI2 analysis. The use of SORM is of course possible and may be necessary for strongly non-linear limit state surfaces. In this case, the reliability index $\beta_{\mathrm{SORM}}=$ $-\Phi^{-1}\left(P_{f, \text { SORM }}\right)$ should be used in Eq. (16).

The simulations of limit state functions including vector processes is currently in progress, as well as the application of the approach to rectangular renewal wave processes and the combination of both kinds of processes in order to deal with problems involving intermittent processes.

Finally, due to the efficiency of the PHI2 method, industrial applications involving finite element analysis of the structure may be considered in the context of timevariant reliability.

\section{References}

[1] Breitung K. Asymptotic approximations for the outcrossing rates of stationary vector processes. Stochast Process Appl 1988;13:195-207.

[2] Schall G, Faber MH, Rackwitz R. The ergodicity assumption for sea states in the reliability estimation of offshore structures. J Offshore Mech Arctic Engng 1991;113:241-6.

[3] Engelung S, Rackwitz R, Lange C. Approximations of first passage times for differentiable processes based on higher order threshold crossings. Probab Engng Mech 1995;10(1):53-60.

[4] Rackwitz R. Computational techniques in stationary and nonstationary load combination-a review and some extensions. J Struct Engng 1998;25(1):1-20.

[5] Hagen O, Tvedt L. Vector process out-crossing as parallel system sensitivity measure. J Engng Mech, ASCE 1991;121(10):2201-20.

[6] Li CC, Der Kiureghian A. In: Lemaire M, Favre J-L, Mébarki A, editors. Mean out-crossing rate of nonlinear response to stochastic input. Proceedings of the Seventh International Conference on Applications of Statistics and Probability (ICASP) in Civil Engineering and Risk Analysis, Paris, France; 1995. p. 295-302.

[7] Li CC, Der Kiureghian A. In: Cooke R, Mendel M, Vrijling H, editors. Mean out crossing of nonlinear response to stochastic input. Engineering probabilistic design and maintenance for flood protection. Dordrecht, The Netherland: Kluwer Academic Publishers; 1997. p. $173-6$

[8] Der Kiureghian A, Li CC. In: Frangopol D, Corotis R, Rackwitz R, editors. Nonlinear random vibration analysis through optimization. Reliability and optimization of structural systems. Proceedings of the Seventh IFIP WG 7.5 Working Conference on Reliability and 
Optimization of Structural Systems. UK: Pergamon Press; 1996. p. 197-206.

[9] Der Kiureghian A. The geometry of random vibrations and solutions by FORM and SORM. Probab Engng Mech 2000;15(1):81-90.

[10] Vijalapura P, Conte J, Meghella M. In: Melchers R, Stewart M, editors. Time-variant reliability analysis of hysteretics SDOF systems with uncertain parameters and subjected to stochastic loading, ICASP8, vol. 1. Amsterdam: Balkema; 2000. p. 827-34.

[11] Andrieu C, Rachad A, Mitteau J-C, Lemaire M. Evaluation d'une structure au cours de sa dégradation dans le temps. Revue Française de Génie Civil, vol. 6. Paris: Hermès; 2002. p. 469-77.

[12] Andrieu C, Lemaire M, Sudret B. The PHI2 method: a way to assess time-variant reliability using time-invariant reliability tools. Proceedings of the European Safety and Reliability Conference ESREL'02, March. Lyon: ISdF; 2002. p. 472-9.

[13] Andrieu-Renaud C. Fiabilité mécanique des structures soumises à des phénomènes physiques dépendant du temps, $\mathrm{PhD}$ Thesis, Université Blaise Pascal, Clermont-Ferrand, France; 2002.

[14] Sudret B, Defaux G, Andrieu C, Lemaire M. Comparison of methods for computing the probability of failure in time-variant reliability using the outcrossing approach. In: Spanos PD, Deodatis G, editors. Proceedings of the Fourth International Conference on Computational Stochastic Mechanics (CSM4). Rotterdam: Millpress; 2003. p. 609-18.

[15] RCP Gmbh, COMREL and SYSREL user's manual, Munich; 1998.

[16] Bolotin VV. Wahrscheinlichkeitsmethoden zur Berechnung von Konstruktionen. Berlin: VEB Verlag für Bauwesen; 1981.

[17] Shinozuka M. Probability of failure under random loading. J Engng Mech Div, ASCE 1964;90(EM5):147-71.

[18] Rice SO. Mathematical analysis of random noise. Bell Syst Technol J 1944;32:282-332.

[19] Rice SO. Mathematical analysis of random noise. Bell Syst Technol J $1945 ; 33: 46-156$.
[20] Belayev YK. On the number of exits across the boundary of a region by a vector stochastic process. Theor Probab Appl 1968;13:320-4.

[21] Cramer H, Leadbetter MR. Stationary and related processes. New York: Wiley; 1967.

[22] Breitung K, Rackwitz R. Nonlinear combinaison of load processes. J Struct Mech 1982;10(2):145-66.

[23] Abdo T, Rackwitz R. A new $\beta$-point algorithm for large time invariant and time-variant reliability problems. Reliability and optimization of structures. Proceedings of the Third WG 7.5 IFIP Conference, Berkeley; 1990.

[24] Bryla P, Faber MH, Rackwitz R. Second order methods in timevariant reliability. Proceedings of the OMAE'91, vol. II; 1991. p. $143-50$.

[25] Hagen O. Threshold up-crossing by second order methods. Probab Engng Mech 1992;7:235-41.

[26] Ditlevsen O, Madsen HO. Structural reliability methods. New York: Wiley; 1996.

[27] Schuëller G. A state-of-the-art report on computational stochastic mechanics. Probab Engng Mech 1997;12(4).

[28] Sudret B, Der Kiureghian A. Stochastic finite element methods and reliability. A state-of-the-art report, Report No. UCB/SEMM-2000/ 08, University of California Berkeley; 2000.

[29] Li CC, Der Kiureghian A. Optimal discretization of random fields. J Engng Mech 1993;119(6):1136-54.

[30] Andrieu C, Lemaire M, Sudret B. Time-variant reliability using the PHI2 method: principles and validation by Monte Carlo simulation. In: Der Kiureghian A, Madanat S, Pestana J-M, editors. Proceedings of the Ninth International Conference on Applications of Statistics and Probability in Civil Engineering (ICASP9). Rotterdam: Millpress; 2003. p. 27-34.

[31] BPEL. Règles techniques de conception et de calcul des ouvrages et constructions en béton précontraint suivant la méthode des états limites, Eyrolles; 1991. 